\title{
Comparative Analysis of Modern Methods of Psychotherapy for Patients with Borderline Personality Disorder
}

\author{
Oleksandr AVRAMCHUK \\ Department of Clinical Psychology, Ukrainian Catholic University, Lviv, Ukraine \\ avramchuk.md@ucu.edu.ua \\ Oksana HLYVANSKA \\ Lviv Regional Clinical Psychiatric Hospital, Lviv, Ukraine \\ ksenja_g@ukr.net
}

\begin{abstract}
This literature review aims to evaluate the evidence in the extant literature of practical and evidence-based psychotherapy methods for overcoming personality disorders and to compare them with one another. For a systematic review, we used the following databases: ScienceDirect, ResearchGate, Cochrane, PsyNet, CrossRef, PubMed. As a result, 33 articles were selected. Dialectic Behavioral Therapy (DBT), Mentalization-based Therapy (MBT) and Schema-focused Therapy (SFT) have better evidence-based efficacy in the context of therapeutic effects on reducing the severity of diagnostic criteria and comorbid states. In the context of application in an outpatient setting the highest positive effect and compliances are demonstrated by DBT and SFT. The majority of trials of DBT and TFP showed the effectiveness of psychotherapy with a treatment duration of at least 12 months. The longest term without recurrence of comorbid states and drop out (36 months) was observed with the use of Transfer-focused Psychotherapy (TFP) and SFT, which in the context of the long-term therapeutic effect may give people benefits along with other therapies. The present review shows initial evidence that long-term psychotherapy can be a useful and evidence-based intervention for people with borderline personality disorder. These results provide promising evidence to support people who suffer from a personality disorder. DBT, MBT, and SFT can be proposed as methods of the first line as a nonpharmacological intervention.
\end{abstract}

Keywords: psychiatry; psychology; mental health; mental illness; personality;

JEL Codes: I12

\section{Background}

Personality disorders have a significant impact on the lives of not only those who have the disorders but also on those who surround them. Personality disorders have been identified as widespread in the global population among all mental health disorders. One of the most studied is borderline personality disorder (BPD) according to DSM-V or emotionally unstable personality disorder (ICD-10).

In the United Kingdom, according to studies from the late 1980s and early 2000s published in the British Journal of Psychiatry in April 2006, the prevalence of personality disorders according to DSM-IV criteria was $4.4 \%$, of which borderline personality disorder (BPD) was $0.7 \%$ (Coid et al., 2006). According to the Ministry of Defense of Great Britain (2008), 9\% of the population have personality disorders, among which the borderline personality disorder has a rate of $1 \%$ (Christmas, 2008). The author points out that $75 \%$ of people who have this disorder are women. The same ratio of gender prevalence is noted in the United States (National Alliance of Mental Illness, 2017), although some studies cite a prevalence of $80 \%$ (Paris, 2005). In contrast, the National 
Alliance of Mental Health (USA) believes that among men BPD has the same frequency as among women; however, in men, it is often misdiagnosed as depression or PTSD (National Alliance of Mental Illness, 2017).

According to the 2001-2002 research statistics published in August of 2004 at Science Daily in the USA, the prevalence of personality disorders according to DSM-IV criteria was $14.8 \%$ of adult Americans (National Institutes of Health, 2004). However, schizotypal, borderline, and narcissistic personality disorders were excluded from this study since the presence of many symptoms needed to be established for their diagnosis. The National Institute of Mental Health in the USA in 2007 notices that approximately 9.1\% of adult Americans have a personality disorder, BPD - 1,4-1,6\% (Lenzenweger et al., 2007). However, it is believed that the prevalence of BPD amounts to $5.9 \%$ due to misdiagnosis (National Alliance of Mental Illness, 2017).

According to WHO studies (2009), the prevalence of personality disorders in the world, according to the criteria of DSM-IV, is $6.1 \%$, and disorders of cluster $\quad-1.5 \%$ and every year this indicator tends to increase (Huang et al., 2009). Unfortunately, there is no reliable data on the prevalence of personality disorders in Ukraine, but we believe that it may reach the average for Western Europe according to the WHO data - 2.4\% (Huang et al., 2009).

The general characteristics of BPD include a significant degree of instability in various personal spheres of human life, along with distorted perceptions of others or their person. Also, sufferers with BPD have interpersonal maladjustment and problems in developing good relationships with society, so they are usually stigmatized as "hard people". As a result, they may commit acts of self-harmed or make suicide attempts during periods of severe depression or episodes of explosive anger and rage.

On the background of strong emotional instability transient psychotic symptoms sometimes are noted (National Collaboration Center for Mental Health, 2009). They manifest in short-term episodes of mania and visual-auditory hallucinations, which requires the necessity to differentiate them from other comorbid disorders, including schizophrenia (National Institute for Health and Care Excellence, 2009). In the family of such persons sometimes there are suicide attempts among their members. Close people, having done everything they could and without having achieved the result to help these people in their problem behaviour, send them for help to doctors.

People who have personality disorders require significant support and psychotherapeutic assistance. Birgit A. Völlm and colleges from the Forensic Psychiatric Unit of the Department of Psychiatry, University of Nottingham (UK) noted that in the treatment of personality disorders, between psychotherapy and psychopharmacology, the priority should be given to the first one (Völlm et al., 2012). Robert S. Biskin from the Institute of Public and Family Psychiatry noted that psychotherapy is a key component in the treatment of personality disorder, as it significantly reduces the symptoms, changes that are usually preserved over an extended period (Biskin and Paris, 2012).

At the same time, people with BPD have problems with regression during therapy (Zanarini, 2009) and so they tend to show a low level of the maintenance of the therapeutic alliance with psychotherapist (Jørgensen et al., 2013). Additionally, this work can be complicated by provoking those who are trying to help them with a countertransference reaction (Zanarini, 2009).

However, many studies point to a significant improvement in patients with personality disorders after undergoing therapy. After 18 months of treatment, 52\% of patients no 
longer met the full list of criteria for personality disorders (Perry et al., 1999). For all clusters of personality disorders, the American Psychiatric Association recommends applying: 1) psychoanalytic/psychodynamic therapy; 2) dialectic-behavioural therapy; 3) cognitive-behavioural therapy; 4) group therapy; 5) psychoeducation for illness, and ways of coping and the course of its treatment (Parekh, 2016). The most studied are dialecticbehavioural therapy, transfer-focused therapy, metallization-based treatment, and a system for training for emotional prevention and problem solving (STEPPS) (Gunderson, 2011; Biskin and Paris, 2012).

Professor of Psychology at the Harvard School of Boston, M. Zanarini in his article "Psychotherapy of Borderline Personality Disorder" (2009), indicated four comprehensive psychosocial methods of treatment for BPD (Zanarini, 2009). Two of these therapies are considered as psychodynamic: mentalization-based treatment and transfer-focused therapy. The other two of the four are cognitive-behavioural: dialecticbehavioural therapy and scheme-focused therapy.

This literature review aims to evaluate the evidence in the extant literature of practical and evidence-based psychotherapy methods for overcoming personality disorders and compare them with one another. It was hypothesized that for the reduction of diagnostic signs of BPD, to improve quality of life and influence on comorbid states, the most evidence-effective is the eclectic approach, such as schema-focused therapy or mentalization-based treatment.

\section{Methods and Organization of Research}

The research on the effectiveness of modern psychotherapy methods was conducted using PRISMA (Preferred Reporting Items for Systematic Reviews and Meta-analyses) recommendations, which were created in 2006 based on the QUOROM standard (Liberati et al., 2009).

\subsection{Study Eligibility}

Eligible studies included: 1) an adult sample, with a mean age of 18 years or older; 2) a clinical diagnoses of borderline personality disorder; 3) analysis of the impact of mentalization-based treatment, transfer-focused psychotherapy, dialectical behavioural therapy, cognitive behavioural therapy, and schema-focused therapy on the borderline personality disorder; 4) randomized controlled trial; controlled clinical trial or longitudinal study; and 5) publication in English.

Qualitative studies, reviews, editorials, and case studies/case series were excluded. Studies explicitly considering other personality disorders were also excluded from this review.

\subsection{Search strategy}

For this systematic review, we used the following databases: ScienceDirect, ResearchGate, Cochrane, PsyNet, CrossRef, PubMed. The search was conducted based on materials from February 1990 to February 2018 and used the following search terms: borderline personality disorder, psychotherapy of personality disorders, mentalizationbased treatment (MBT), transfer-focused psychotherapy (TFP), dialectic-behavioural 
therapy (DBT), schema-focused therapy (SFT), cognitive-behavioural therapy (CBT), effectiveness.

Search lines were used consistently in all databases in which the search was performed; excerpts were not used. When reviewing the articles, additional searches were done on the corresponding citations. There was no contact with the authors for research.

\subsection{Description of the sampling procedure}

Among the 309 articles that were found on the resources, we excluded duplicate reports, and articles that match the exclusion criteria. As a result, we selected 33 studies. For further detailed assessment, the results were synthesized narratively. Studies were grouped into categories on psychotherapeutic approaches. These include: studies on the effectiveness of dialectical behavioural therapy of borderline personality disorder $(\mathrm{k}=$ 12); studies that assess effectiveness of mentalization-based treatment of borderline personality disorder $(\mathrm{k}=5)$; studies that evaluate the efficacy of transfer-focused psychotherapy of borderline personality disorder $(\mathrm{k}=5)$; studies that assess effectiveness of schema-focused therapy of borderline personality disorder $(\mathrm{k}=4)$ and effectiveness of cognitive-behavioural therapy of border personality disorder $(\mathrm{k}=7)$.

To optimize the literature data obtained and to compare them better, we used the following aspects:

- reducing the severity of diagnostic criteria for BPD;

- the focus of therapy on suicide / self-harming behaviour;

- orientation to the comorbid states;

- improving the quality of life and social adaptation;

- opportunity to use in an outpatient setting;

- the duration of therapy to achieve its greatest effectiveness and level of treatment drop-out.

These aspects were chosen for structuring the data on therapy, because of their expressing quantitative and qualitative indicators of the effectiveness of therapy. Although several elements have been selected, they are not all-embracing. Therefore, complementing these aspects is a prospect for further research.

\section{Results and Discussion}

The present review synthesizes literature exploring the effectiveness of five psychotherapy approaches for borderline personality disorder. All 33 identified studies showed a positive association between reducing the psychosocial distress and interpersonal maladjustment of people with BPD and psychotherapeutic interventions. However, comparing the effects of different psychotherapies on BPD was limited, making the direction of which interventions are more effective unclear and causality impossible to establish. The findings do suggest that certain experiences in psychotherapy play a key role in overcoming this mental disorder.

\subsection{Reducing the severity of diagnostic criteria for BPD}

According to the data obtained, 23 studies aimed at assessing the effectiveness of the psychotherapeutic interventions by decreasing the severity of diagnostic signs of BPD. DBT (Koons et al., 2001; Bohus et al., 2004; McMain et al., 2009), SFT (Giesen-Bloo et al., 2006, van Asselt et al., 2008, Nadort et al., 2009), and MBT (Bateman and Fonagy, 
1999, 2009; Rossouw and Fonagy, 2012; Jørgensen et al., 2013, Laurenssen et al., 2018) have better evidence-based efficacy in the context of therapeutic effects on reducing the severity of diagnostic criteria as per the DSM-IV. For assessment authors usually used Structured Clinical Interviews for the DSM-IV, versions I and II or other semi-structured clinical interviews relevant and recommended by the American Psychiatry Association.

Research by J.M. Farrell et al. (2009) showed that diagnostic criteria were not met after completion of SFT in $94 \%$ of subjects. The results of the research conducted on DBT also indicated a significant reduction of anxiety (Neacsiu et al., 2014; Harned et al., 2014), improved impulsivity and anger regulation (Linehan et al., 1994; Clarkin et al., 2007).

It should be noted that the data from the analysis was not generalized to the men's population or individuals with comorbid schizophrenia, bipolar disorder, or other severe mental disorders. Another issue: does the therapeutic effect of reducing the severity of the diagnostic criteria will be watched in patients who dropped out and returned in psychotherapeutic settings.

\subsection{The direction of therapy for suicide / self-harming behaviour}

According to the results review, the number of DBT studies that set the primary goal of detecting the impact of psychotherapy on suicide / self-harming behaviour was $44 \%$ from all articles. All research on DBT selected for review, except for A.D. Neacsiu and colleges (2014), showed a statistically significant reduction rate of suicide attempts, parasuicide or deliberate self-harm in BPD.

The same efficiency was demonstrated using the CBT techniques. Studies selected for review of the application of CBT showed the effect of these interventions on reducing the frequency and severity of intentional self-harm (Evans et al., 1999; Weinberg et al., 2006). The work of MacLeod et al. (1998) did not show a positive effect of CBT on reducing the suicidal / self-harmful behaviour, but there it was a reduction in parasuicidal thinking through improved positive thinking about the future. According to these studies, the duration of therapy should be at least six months to achieve its highest effectiveness. Finally, the level of application for a psychiatric clinic for these complaints above and after the treatment was decreased.

A statistically significant reduction in the level of suicidality / parasuicidal activity was shown by studies of mentalization-based treatment and schema-focused therapy. Studies have shown achieving the efficiency level of interpersonal functioning and social adaptation along with a decrease in the level of suicidal / self-harmful behaviour. Therefore, it can be assumed that effective psychotherapy interventions that focus on affective, identity, and interpersonal disturbances have the reverse correlation with the level of such behavior. The findings of Farrell et al. (2009) partly confirmed this hypothesis and Rossouw et al. (2012) suggested that increasing the level of interpersonal functioning might correlate with the level of reduction of self-harmful behaviour. However, to confirm this hypothesis, further research is needed.

\subsection{Orientation to the comorbid states}

Since BPD has high comorbidity (National Collaboration Center for Mental Health, 2009), it is often possible to identify cases of its combination with anxiety disorders, mood disorders, eating disorders, somatoform disorders, and drug-related disorders (Zanarini et al., 1998). Often symptoms of bipolar disorder are generally perceived as symptoms of BPD (May et al., 2016). Conceptual confusion and finding this personality disorder at the "edge" of other personality disorders also makes it diagnostically 
comorbid with the disorders as mentioned earlier (National Institute for Health and Care Excellence, 2009).

Among the articles selected for the survey, it was noted that most often the initial focus of psychotherapy is depression. Most of the studies on DBT (Koons et al., 2001; Bohus et al., 2004; Linehan et al., 2006; Clarkin et al., 2007; McMain et al., 2009) showed a decrease in depression level after the completion of the course of therapy. Similar results are seen by using of the MBT (Bateman and Fonagy, 1999, 2009; Rossouw et al., 2012; Jørgensen et al., 2013; Laurenssen et al., 2018).

Such some studies might be as evidence of high comorbidity of depression with BPD, and as the effectiveness of psychotherapy in the treatment of depression. Also, it can be an indication that depression can be a significant factor in increasing suicide in BPD. Three studies on DBT (Linehan et al., 1999; Verheul et al., 2003; Harned et al., 2014) in our review were related to addiction to substance abuse and PTSD.

\subsection{Improving the quality of life and social adaptation}

The relevance of the problem of personality disorders prevalence is not only its devastating effects on the lives of patients and their relatives but also on society around them. At the same time, the prevalence in different countries, which have excellent cultural and social background (de Girolamo, 1995) is of interest. Regarding outcomes, it was determined that $24 \%$ of the total number of studies that have shown positive effects on the quality of life patients with a borderline personality disorder is related to DBT (8 from 12 studies of DBT including for review). $12 \%$ of studies reviewed focused on SFP and MBT, while $9 \%$ focused on TFP, and 3\% on and CBT. Another 11 studies didn't use this goal aspect for their research, and two studies of CBT showed no significant changes of interpersonal and psychosocial functioning (Tyrer et al., 2003; Davidson et al., 2006) compared to treatment as usual.

The aim of mentalization-based treatment is to increase the individual's capacity to evoke and reflect on one's own experience to make inferences about behaviour in oneself and others. MBT leads to improvement of affective regulation, reduction of suicidal and selfharmful behaviour, improvement of interpersonal relationships, etc. (Rossouw et al., 2012; Jørgensen et al., 2013; Laurenssen et al., 2018). Also, a study by Bateman and Fonagy (2009) on the efficacy of MBT has demonstrated a reduction in the number of drugs used by participants who received and completed treatment. In our opinion, this can be described as one of the basic aspects of the effectiveness of this therapy.

As noted by Giesen-Bloo et al. (2006), SFT and TFP address BPD at the level of personality and improved quality of life. Namely, their primary goals don't direct the reduction of self-destructive psychopathological dysfunction of BPD as the DBT mentioned earlier, CBT or even MBT. In schema-therapy, the goal is to change the person's internal structure, which occurs due to work with dysfunctional schemas and formation of therapist's "reparenting" attitude on attachment issues of patients with BPD. Giesen-Bloo et al. (2006) found that after three years of courses of SFT more patients recovered and showed substantial clinical improvement compared to patients in TFP. Levy et al. (2006) and Doering et al. (2010) showed that TFP helped people with BPD improve significantly in reflective function, attachment style, and personality organization. 


\subsection{Opportunity to use in and outpatient setting}

People with BPD might have different levels of behavioural and emotional problems. Some of them can maintain relationships and professional activities. Sometimes they have interpersonal maladjustment that they can manage themselves or with the support of relatives, without severe consequences such as increasing the frequency of drug use (both prescribed and illegal) or suicidal attempts. The other part, which is more severe, is emotional distress. On the background of repeated crises with impulsive-aggressive or even self-harmful behaviour, individuals often require emergency medical care, and they become patients of psychiatric hospitals (NICE, 2009). However, the J. M. May and colleagues study pointed out that outpatient services for people with BPD have a higher cost in comparison with separate major depressive disorder or other personality disorders (May et al., 2016).

Five studies of DBT (Linehan et al., 1991, 2006; Koons et al., 2001; McMain et al., 2009; Carter et al., 2010) showed a decrease in the rate of inpatient care after completing the course of psychotherapy, and along with this no studies were found that showed no change or increases in the inpatient level. That might indicate a positive effect of therapy on reducing the frequency of some hospital admissions and time spent as an inpatient during a crisis. In considering other DBT studies, the authors talked about the possibility of implementation in outpatient settings. The successful application of DBT in outpatient settings has been demonstrated in studies by Verheul et al. (2003) and Neacsiu et al. (2014).

In studies by Bateman and Fonagy (2009) and Rossouw and Fonagy (2012) MBT interventions implemented in outpatient settings have demonstrated their success. According to the authors, this is not only a positive indicator of the therapeutic effectiveness of therapy but also its cost-effectiveness. The study by Laurenssen et al. (2018) was conducted in a day hospital. According to the outcomes, it confirms the efficacy of the use of this therapy in the outpatient network.

Successful implementation of TFP measures in outpatient settings was demonstrated in a study by Doering et al. (2010), Levy et al. (2006), Clarkin et al. (2007), and Fischer-Kern et al. (2015) recommended it for implementation. The results of SFP efficacy studies in outpatient settings have demonstrated in the works of Giesen-Bloo et al. (2006) and van Asselt et al. (2008).

\subsection{The duration of therapy to achieve its greatest effectiveness}

Most trials of DBT and TFP showed the effectiveness of psychotherapy with a treatment duration of at least 12 months. For all others, this term was at least 18 months. However, the study of Bohus et al. (2004) showed the effectiveness of DBT in the hospital from the 3rd month of psychotherapy course, and the study of Koons et al. (2001) and Carter et al. (2010), which took place in an outpatient setting, showed the reduction of psychopathological dysfunction from the 6th month. That date can significantly affect the choice of treatment for some patients. Linehan et al. (2006) and Neacsiu et al. (2014) recorded the most significant effect of therapy after 24 months. At the same time, the extended term of the therapy itself did not exceed 12 months. The most extended term without relapse of comorbid states and drop out (36 months) was observed with the use of TFP and SFT (Giesen-Bloo et al., 2006, van Asselt et al., 2008). These results can give people with BPD more benefits in the context of a long-term therapeutic effect compared with other therapies. 
It should be noted that in most studies, DBT and MBT had a sophisticated form, a combination of individual and group, while TFT, SFT, and CPT studies were conducted in an individual format.

\subsection{Level of drop-out of therapy}

The present review demonstrates the importance of patients with BPD continuing the course of therapy and its correlation with the recovery of patients with BPD. Many patients throw the therapy out of reach.

According to the results in the treatment process with the use of DBT, the reduction of drop-out level was established and amounted to 22-39\% (Linehan et al., 1999; Koons et al., 2001; Bohus et al., 2004; Harned et al., 2014). In another study, Laurenssen et al. (2018) demonstrated 9\% drop-out from therapy after the mentalization-based treatment in the hospital. However, in a survey by Jørgensen et al. (2013) in the outpatient setting, the drop-out level was $32 \%$, while, as noted by the authors, there was no significant difference between the experimental and control groups. A study by Doering et al. (2010) observed a relatively high level of drop-out of therapy when using TFT (38.5\%) - higher than DBT (25\%) and SFP (26.7\%). However, these studies showed significantly lower drop-out rates compared with treatment as usual. The authors of this study suggest that lower drop-out rates may be due to a positive picture of coverage by insurance companies of all appeals to psychotherapists. Research by Farrell et al. (2009) showed the level of drop-out to be zero when using SFP compared with the control group of treatment as usual. However, such a low level of drop-out of therapy may be due to a small sample experimental group $(n=16)$.

That is why we cannot argue that such a low level will be observed with an increase in the number of SFP studies. On the other hand, the direction of therapy can explain the positive effect. In Schema-focused Therapy psychotherapist work with dysfunctional schemes which can be involved in the strategy of avoiding and abandoning the patient's treatment.

\section{Review limitations}

The findings of this review must be understood in the context of several limitations. The present analysis includes the low number of articles on the effectiveness of mentalizationbased treatment, transfer-focused psychotherapy, schema-focused therapy and other approaches in comparison with dialectic-behavioural therapy. The majority of studies that reviewed the effect of CBT did not demonstrate the reduction in the severity of diagnostic criteria for BPD, changes in interpersonal functioning or social adaptation nor was the decrease in the level of drop-out of therapy mentioned.

The reviews included were limited to studies published in English, and as such may have excluded several relevant studies from other languages and cultures. Studies exploring borderline personality disorder included $75 \%$ and higher women participants. We have mentioned that samples including men may have altered the conclusions.

We understood that studies show there is a limited chance that people with BPD will be cured from this personality disorder, and psychotherapeutic interventions focus on maintaining and improving life quality. The majority of studies that were reviewed write about follow-up effects of psychotherapy beyond 12 months, which also limited our ability to compare the efficacy of treatment modalities. 


\section{Conclusions}

In conclusion, the current study suggests that psychotherapy is an effective approach in the treatment of BPD. The results of the article review can be used by psychotherapists and other specialists of mental health services when choosing the direction of therapy for borderline personality disorder. Also, the results can be used for psychoeducational intervention for a patient with this disorder, and their relatives.

DBT, SFT, and MBT could be proposed as methods of the first line as a nonpharmacological intervention for people with BPD. They have better evidence-based efficacy in the context of therapeutic effects on reducing the severity of diagnostic criteria and comorbid states. Namely, DBT showed straightforward direction and targeted strategies for decreasing severity self-destructive psychopathologic dysfunction of BPD. CBT interventions and management, which oriented on changing parasuicidal thinking, also can be useful for relapse prevention. At the same time, the studies of mentalizationbased treatment and schema-focused therapy showed an increase in the level of interpersonal functioning and social adaptation, along with a decrease in the level of suicidal / self-harmful behaviour.

In the context of application in outpatients setting, which is a more recommended direction given the focus of modern medical protocols and manuals, DBT showed better evidence of efficacy. According to the analysis of the studies, the most positive effects and compliances are demonstrated by DBT and SFT.

Most trials of DBT and TFP showed the effectiveness of psychotherapy with a treatment duration of at least 12 months. For all others, this term was at least 18 months. Three trials (Koons et al., 2001, Bohus et al., 2004, Carter et al., 2010) showed the effectiveness of DBT in the third to sixth months, which can significantly affect the choice of therapy for some patients. At the same time, the use of TFP and SFT was observed in the most extended term without recurrence of comorbid states and drop out (36 months). The longterm therapeutic effect of TFP and SFT can give people with BPD more benefits for improved quality of life compared with other psychotherapies.

\section{References:}

Bateman, A., Fonagy, P. (1999): Effectiveness of Partial Hospitalization in the Treatment of Borderline Personality Disorder: A Randomized Controlled Trial. The American Journal of Psychiatry, 156, 1563-1569. http://doi.org/10.1176/ajp.156.10.1563

Bateman, A., Fonagy, P. (2009): Randomized Controlled Trial of Outpatient Mentalization-Based Treatment versus Structured Clinical Management for Borderline Personality Disorder. The American Journal of Psychiatry, 166(12), 1355-1364. http://doi.org/10.1176/appi.ajp.2009.09040539

Biskin, R.S., Paris, J. (2012): Management of borderline personality disorder. CMAJ, 184(17), 1897-1902. http://doi.org/10.1503/cmaj.112055

Bohus, M., Haaf, B., Simms, T., Limberger, M.F. (2004): Effectiveness of inpatient dialectical behavioral therapy for borderline personality disorder: a controlled trial. Behaviour Research and Therapy, 42(5), 487-499. http://doi.org/10.1016/S0005-7967(03)00174-8

Carter, G. L., Willcox, C. H., Lewin, T. J., Conrad, A. M., Bendit, N. (2010): Hunter DBT project: randomized controlled trial of dialectical behaviour therapy in women with borderline personality disorder. The Australian and New Zealand Journal of Psychiatry, 44(2), 162-173. http://doi.org/10.3109/00048670903393621

Christmas, D. (2008): Synopsis of Causation: Personality Disorder, Ministry of Defence, 1-24. 
Clarkin, J. F., Levy, K. N., Lenzenweger, M. F., Kernberg O. F. (2007): Evaluating Three Treatments for Borderline Personality Disorder: A Multiwave Study. The American Journal of Psychiatry 164(6), 922-928 http://doi.org/10.1176/ajp.2007.164.6.922

Coid, J., Yang, M., Tyrer, P., Roberts, A., Ullrich, S. (2006): Prevalence and correlates of personality disorder in Great Britain. The British Journal of Psychiatry, 188(5), 423-431 http://doi.org/10.1192/bjp.188.5.423

Davidson, K., Norrie, J., Tyrer, P., Gumley, A., Tata, P., Murray, H., Palmer, S. (2006): The effectiveness of cognitive behavior therapy for borderline personality disorder: results from the borderline personality disorder study of cognitive therapy (BOSCOT) trial. Journal of Personality Disorders, 20(5), 450-465. http://doi.org/10.1521/pedi.2006.20.5.450

De Girolamo, G., Reich, J.H. \& WHO (1995): сстройств личности. G.de Girolamo, J.H.Reich. WHO. http://www.who.int/iris/handle/10665/89936

Doering, S., Hörz, S., Rentrop, M., Fischer-Kern, M., Schuster, P. et al. (2010): Transferencefocused psychotherapy v. treatment by community psychotherapists for borderline personality disorder: randomised controlled trial. The British Journal of Psychiatry, 196, 389-395. http://doi.org/10.1192/bjp.bp.109.070177

Evans, K., Tyrer, P., Catalan, J., Schmidt, U., Davidson, K., Dent, J., Tata, P., Thornton, S., Barber J. (1999): Manual-assisted cognitive behavior therapy (MACT): a randomized controlled trial of a brief intervention with bibliotherapy in the treatment of recurrent deliberate self-harm. Psychological Medicine, 29, 19-25.

Farrell, J. M., Shaw, A., Webber, M. A. (2009): A schema-focused approach to group psychotherapy for outpatients with borderline personality disorder: A randomized controlled trial. Journal of Behavior Therapy and Experimental Psychiatry, 40(2), 317-328. http://doi.org/10.1016/j.jbtep.2009.01.002

Fischer-Kern, M., Doering, S., Taubner, S., Hörz, S., Zimmermann, J., Rentrop, M., Schuster, P., Buchheim, P., Buchheim, A. (2015): Transference-focused psychotherapy for borderline personality disorder: change in reflective function. The British Journal of Psychiatry, 207(2), 173-174. http://doi.org/10.1192/bjp.bp.113.143842

Giesen-Bloo J., R. van Dyck, P. Spinhoven, van Tilburg W. et al. (2006): Outpatient psychotherapy for borderline personality disorder: randomized trial of schema-focused therapy vs transference-focused psychotherapy. Archieves of General Psychiatry, 63(6), 649-658. http://doi.org/10.1001/archpsyc.63.6.649

Gunderson, J. G. (2011): Clinical practice. Borderline Personality Disorder. The New England Journal of Medecine, 364, 2037-2042. http://doi.org/10.1056/NEJMcp1007358

Harned, M. S., Korslund, K. E., Linehan, M. M. (2014): A pilot randomized controlled trial of Dialectical Behavior Therapy with and without the Dialectical Behavior Therapy Prolonged Exposure protocol for suicidal and self-injuring women with borderline personality disorder and PTSD. Behaviour Research and Therapy, 55, 7-17. http://doi.org/10.1016/j.brat.2014.01.008

Huang, Y., Kotov, R., de Girolamo, G., Preti, A., Angermeyer, M., Benjet, C., ... Kessler, R.C. (2009): DSM-IV personality disorders in the WHO World Mental Health Surveys. The British Journal of Psychiatry, 195(1), 46-53. http://doi.org/10.1192/bjp.bp.108.058552

Jørgensen, C. R., Freund, C., Bøye, R., Jordet, H., Andersen, D., Kjølbye, M. (2013): Outcome of mentalization-based and supportive psychotherapy in patients with borderline personality disorder: a randomized trial. Acta Psychiatrica Scandinavica, 127, 305-317. http://doi.org/10.1111/j.1600-0447.2012.01923.x

Koons, C. R., Robins, C. J., Tweed, J. L., Lynch, T. R., Gonzalez, A. M., Morse, J. Q., ... Bastian, L.A. (2001): Efficacy of dialectical behavior therapy in women veterans with borderline personality disorder. Behavior Therapy, 32(2), 371-390. http://dx.doi.org/10.1016/S00057894(01)80009-5 
Laurenssen, E., Luyten, P., Kikkert, M. J., Westra, D., Peen, J., Soons, M. B. J., van Dam, A.-M., ... Dekker, J. (2018): Day hospital mentalization-based treatment v. specialist treatment as usual in patients with borderline personality disorder: randomized controlled trial. Psychological Medicine, 26, 1-8. http://doi.org/10.1017/S0033291718000132

Lenzenweger, M.F., Lane, M.C., Loranger, A.W., \& Kessler, R.C. (2007): DSM-IV personality disorders in the National Comorbidity Survey Replication. Biological Psychiatry, 62(6), 553-564. http://doi.org/10.1016/j.biopsych.2006.09.019

Levy, K. N., Meehan, K. B., Kelly, K. M., Reynoso, J. S., Weber, M., Clarkin, J.F., Kernberg O. F. (2006): Change in Attachment Patterns and Reflective Function in a Randomized Control Trial of Transference-Focused Psychotherapy for Borderline Personality Disorder. Journal of Consulting and Clinical Psychology, 74(6), 1027-1040. http://doi.org/10.1037/0022006X.74.6.1027

Liberati, A., Altman, D. G., Tetzlaff, J., Mulrow, C., Gøtzsche, P. C., Ioannidis, J., Clarke, M., Devereaux, P. J., Kleijnen, J., Moher, D. (2009): The PRISMA Statement for Reporting Systematic Reviews and Meta-Analyses of Studies That Evaluate Health Care Interventions: Explanation and Elaboration. PLOS Medicine, 6(7). http://doi.org/10.1371/journal.pmed.1000100 Linehan, M. M., Armstrong, H. E., Suarez, A., Allmon, D., Heard, H.L. (1991): Cognitivebehavioral treatment of chronically parasuicidal borderline patients. Archieves of General Psychiatry, 48(12), 1060-1064.

Linehan, M. M., Tutek, D. A., Heard, H. L., Armstrong, H.E. (1994): Interpersonal outcome of cognitive behavioral treatment for chronically suicidal borderline patients. The American Journal of Psychiatry, 151(12), 1771-1776. http://doi.org/10.1176/ajp.151.12.1771

Linehan, M. M., Schmidt, H., Dimeff, L. A., Christopher, C. J., Kanter, J., Comtois, K. A. (1999): Dialectical Behavior Therapy for Patients with Borderline Personality Disorder and DrugDependence. The American Journal on Addictions, 8(4), 279-292. http://doi.org/10.1080/105504999305686

Linehan, M. M., Comtois, K. A., Murray, A. M., Brown, M. Z. et al. (2006): Two-Year Randomized Controlled Trial and Follow-up of Dialectical Behavior Therapy vs Therapy by Experts for Suicidal Behaviors and Borderline Personality Disorder. Archieves of General Psychiatry, 63, 757-766. http://doi.org/10.1001/archpsyc.63.7.757

May, J.M., Richardi, T.M., Barth, K.S. (2016): Dialectical behavior therapy as treatment for borderline personality disorder. The College of Psychiatric and Neurologic Pharmacists. The Mental Health Clinician, 6(2), 62-67. https://doi.org/10.9740/mhc.2016.03.62

MacLeod, A. K., Tata, P., Evans, K., Tyrer, P., Schmidt, U., Davidson, K., Thornton, S., Catalan, J. (1998): Recovery of positive future thinking within a high-risk parasuicide group: results from a pilot randomized controlled trial. British Journal of Clinical Psychology, 37(4), 371-379.

McMain, S. F., Links, P. S., Gnam, W. H., Guimond, T. (2009): A Randomized Trial of Dialectical Behavior Therapy Versus General Psychiatric Management for Borderline Personality Disorder. The American Journal of Psychiatry, 166(12), 1365-1374. http://doi.org/ 10.1176/appi.ajp.2009.09010039

Nadort, M., Arntz, A., Smit, J. H., Giesen-Bloo, J., Eikelenboom, M., Spinhoven, P., van Asselt, T., Wensing, M., van Dyck, R. (2009): Implementation of outpatient schema therapy for borderline personality disorder with versus without crisis support by the therapist outside office hours: A randomized trial. Behaviour Research and Therapy, 47(11), 961-973. http://doi.org/ 10.1016/j.brat.2009.07.013

National Alliance of Mental Illness (2017): Borderline Personality Disorder. Overview. Available at: https://www.nami.org/Learn-More/Mental-Health-Conditions/Borderline-PersonalityDisorder

National Collaboration Center for Mental Health (2009): Borderline Personality Disorder: the NICE guideline on treatment and management. Available 
at: https://www.nice.org.uk/guidance/cg78/resources/borderline-personality-disorder-recognitionand-management-pdf-975635141317

National Institute for Health and Care Excellence (NICE) (2009): Borderline personality disorder: recognition and management. Clinical guideline [CG78]. Available at: https://www.nice.org.uk/guidance/cg78

Neacsiu, A.D., Lungu, A., Harned, M.S., Rizvi, S.L., Linehan, M.M. (2014): Impact of Dialectical Behavior Therapy versus community treatment by experts on emotional experience, expression, and acceptance in borderline personality disorder. Behaviour Research and Therapy, 53, 47-54. http://doi.org/10.1016/j.brat.2013.12.004

NIH/National Institute On Alcohol Abuse And Alcoholism (2004, August 3): Landmark Survey Reports On The Prevalence Of Personality Disorders In The United States. ScienceDaily. Available at: www.sciencedaily.com/releases/2004/08/040803095121.htm

Parekh, R. (2016): What are Personality Disorders? Personality Disorders. American Psychiatry Association. Available at: https://www.psychiatry.org/patients-families/personalitydisorders/what-are-personality-disorders

Paris, J. (2005): Borderline personality disorder. CMAJ, 172(12), 1579-1583. http://doi.org/10.1503/cmaj.045281

Perry, J.C., Banon, E., Ianni, F. (1999) Effectiveness of Psychotherapy for Personality Disorders. The American Journal of Psychiatry, 156(9), 1312-1321. http://doi.org/10.1176/ajp.156.9.1312

Rossouw, T.I., Fonagy, P. (2012): Mentalization-based treatment for self-harm in adolescents: a randomized controlled trial. Journal of the American Academy of Child and Adolescent Psychiatry, 51(12), 1304-1313. http://doi.org/10.1016/j.jaac.2012.09.018

Tyrer, P., Thompson, S., Schmidt, U., Jones, V., Knapp, M., Davidson, K., Catalan, J., Airlie, J., Baxter, S., Byford, S., Byrne, G. et al. (2003): Randomized controlled trial of brief cognitive behaviour therapy versus treatment as usual in recurrent deliberate self-harm: the POPMACT study. Psychological Medicine, 33(6), 969-976.

van Asselt, A. D., Dirksen, C. D., Arntz, A., Giesen-Bloo, J. H., van Dyck, R., Spinhoven, P., van Tilburg, W. et al. (2008): Out-patient psychotherapy for borderline personality disorder: costeffectiveness of schema-focused therapy v. transference-focused psychotherapy. The British Journal of Psychiatry, 192(6), 450-457. http://doi.org/10.1192/bjp.bp.106.033597

Verheul, R., Van Den Bosch, L. M., Koeter, M. W., De Ridder, M. A., Stijnen, T., van den Brink, W. (2003): Dialectical behaviour therapy for women with borderline personality disorder: 12month, randomised clinical trial in The Netherlands. The British journal of psychiatry, 182, 135140.

Völlm, B. A., Chadwick, K., Abdelrazek, T., Smith, J. (2012): Prescribing of psychotropic medication for personality disordered patients in secure forensic settings. The Journal of Forensic Psychiatry \& Psychology, 23(2), 200-216. http://doi.org/10.1080/14789949.2012.655764

Weinberg, I., Gunderson, J. G., Hennen, J., Cutter, C.J.Jr. (2006): Manual assisted cognitive treatment for deliberate self-harm in borderline personality disorder patients. Journal of Personality Disorders, 20(5), 482-492. http://doi.org/10.1521/pedi.2006.20.5.482

Zanarini, M.C. (2009): Psychotherapy of Borderline Personality Disorder. PubMed Central. Acta Psychiatrica Scandinavica, 120(5), 1-8. http://doi.org/10.1111/j.1600-0447.2009.01448.x 